\title{
Optimization of Sensing Receiver for Cognitive Radio Applications
}

\author{
Hassan Zamat ${ }^{1}$ and Balasubramaniam Natarajan ${ }^{2}$ \\ ${ }^{1}$ IBM Systems and Technology Group, 4660 La Jolla Village Dr., Suite 300, San Diego, CA 92127, USA \\ ${ }^{2}$ Director WiCom Research Group, Department of Electrical Engineering, Kansas State University, Manhattan, KS 66506, USA
}

Correspondence should be addressed to Hassan Zamat, zamat@us.ibm.com

Received 14 February 2009; Revised 26 May 2009; Accepted 8 July 2009

Recommended by R. Chandramouli

\begin{abstract}
We propose an optimized dedicated broadband sensing receiver architecture for use in cognitive radios supporting delay sensitive applications. Specifically, we first reason the need for a dedicated sensing receiver that employs a combination of coarse and fine scanning to reduce sensing time over a large bandwidth. We derive an expression for mean acquisition/detection time as a function of a number of parameters including the number of coarse and fine frequency bins employed. We then determine the optimal number of coarse and fine bins that minimize the overall detection time required to identify idle channels under various system conditions. Using analytical and simulation results, we quantify the dependence of optimal coarse and fine bin selection on system parameters such as (1) size of FFT used in scanning; (2) probability of detection and false alarm of the underlying sensing algorithm; (3) signal-to-noise ratio of the received signal, and (4) expected number of available channels. The primary contribution of this work lies in a practical realization of an optimal broadband sensing receiver.
\end{abstract}

Copyright (c) 2009 H. Zamat and B. Natarajan. This is an open access article distributed under the Creative Commons Attribution License, which permits unrestricted use, distribution, and reproduction in any medium, provided the original work is properly cited.

\section{Introduction}

Cognitive Radios (CRs) promise to address the underutilization of the frequency spectrum-a scarce and precious resource required for wireless communication. CRs are capable of operating as secondary users (SUs) adding limited interference to the primary users (PUs) and other secondary users in a desired band. The key to spurring the wide adoption of CR in the market is a practical realization of the sensing receiver in the cognitive radio. The receiver must have the ability to make fast and accurate decisions on availability (or lack thereof) of a channel. The sensing receiver must actively scan, detect, initiate a communication link, and dynamically adjust its transmission parameter in order to minimize interference with existing users. Technological advances in the recent years have addressed some of the challenges of broadband, frequency agile radios. However, real challenges still persist such as the physical implementation of broadband frequency synthesizers, high sensitivity receivers with high dynamic range [1-4]. In order to address these shortcomings, the CR community is focusing on innovative architectures and algorithms.
Cognitive Radios (CRs) require an accurate assessment of the activities in a desired frequency spectrum in order to determine the availability of idle channels suitable for opportunistic secondary use. Prior research has focused on novel techniques such as centralized network sensing [5], cooperative sensing [6], and the use of statistical methods and game theory [7] to improve sensing time. These techniques are not well suited for practical implementation of CR in time sensitive operations. By utilizing the techniques presented in [5-7], the CR is now dependent on external inputs to make decisions on its operation. With a centralized network sensing, additional costs and delays are introduced by the traffic controller. In the cooperative model, the CR performs energy detection and uses time division slots to communicate with other users. As the number of users increase, the delay may become intolerably long. In our earlier work [8], we propose the use of a dedicated sensing receiver (DSR) that is solely focused on channel sensing and runs in parallel with a main receiver. The key to the DSR is an efficient algorithm that performs spectrum detection and continuously improves the quality of the collected data and decision process. The fast and initial sensing is 
done in the analog domain at the RF or IF frequencies prior to additional processing in the digital domain. We demonstrated that the use of a dedicated sensing receiver (DSR) is necessary and required for fast and reliable sensing in broadband operation. In addition, the overall time delay is also greatly reduced which opens the way for voice operation in cognitive radio. We were able to show that the DSR architecture provides up to a fivefold reduction in total mean time detection.

In this paper, we focus on optimizing the broadband sensing receiver architecture for use in cognitive radios supporting delay sensitive applications. In our proposed DSR model, we use a two-stage sensing technique for performing broadband sensing. Here, we divide the desired bandwidth into coarse bins which are then subdivided into fine bins. After the initial setup, the receiver performs a cursory scan of the coarse bins in search of idle channels. Once idle channels are identified, the receiver then proceeds to a more thorough scan of the channels using improved resolution in order to avoid misdetection or a false alarm (especially when the primary users of the channel are operating at low signal to noise ratio (SNR)). Higher resolution scans require more time to complete the operation. The coarse scan while faster is not as accurate and might lead to a high number of misdetections. Hence, a delicate balance between the faster coarse scan and the more accurate but slower fine scan is needed. Therefore, we first derive an expression for total mean detection time as a function of the number of coarse and fine bins as well as other system parameters such as phase locked loop (PLL) lock time, digital signal processing (DSP) frequency of operation, and received signal to noise ratio. We then determine the optimal values of coarse and fine bins that minimize the total mean detection time. Using both analytical and simulation results, we quantify the effect of various system parameters on the optimal choice of coarse and fine bins. For example, we show that the optimal number for coarse bins decreases with an increase in SNR and the optimal number of fine bins increases with increased interference in the band.

This paper consists of five sections. In Section 2, we present our Dedicated Sensing Receiver architecture, define the channel model, and derive an equation for mean detection time. In Section 3, we optimize the coarse and fine bin size such that our total mean detection time is minimized. The results from optimization are presented in Section 4. Section 5 presents the conclusions and future work.

\section{Dedicated Sensing Receiver}

Although spectrum is overcrowded at frequencies below $3 \mathrm{GHz}$, the utilization drops to less than $0.5 \%$ above $3 \mathrm{GHz}$ [9]. It is not a trivial task to design a broadband and agile radio that operates above $3 \mathrm{GHz}$. The radio design challenges include receiver sensitivity, dynamic range, frequency generation (synthesizers), and other RF impairments $[3,7]$. As the radio cycles through the frequencies of interest, the PLL lock time, becomes a significant contributor to the total scan time. As the frequency step increases, the PLL lock and settling times degrade. Once settled, the receiver could exercise a Periodogram Spectral Estimator (PSE) which makes use of fast Fourier transform (FFT) for spectral detection. FFTs are computationally intensive and the time required to perform the computation is directly proportional to the DSP speed used in the system. If a higher frequency resolution is desired, we require a longer observation time. Hence as the number of FFT points increases, the resolution improves but the scanning time degrades. A compromise between frequency estimation and detection bandwidth is therefore required. In this paper, we proposed a two-stage approach in which a coarse scan with lower number of FFT points is performed on a large bandwidth in search of idle channels. Once the idle coarse channels are identified, a higher number of FFT points are used to perform the fine scan. In order to avoid false alarms and minimize the probability of interfering with a user in the band, the CR must continuously monitor the spectrum for activity of other occupants in the spectrum. Without a radio receiver dedicated to sensing the spectrum, the main receiver is continuously interrupted in order to perform sensing and link maintenance. The interruption and delays are detrimental to time sensitive applications such as video and audio. Based on popular voice Codecs and ITU recommendations [10-12], it is possible to translate the acceptable voice delays to CR delay requirements [8]. For the purposes of this work, we propose a rule of thumb for total time delay between packet transmissions to be less than 20 milliseconds. In [8], we illustrate that it is possible to meet this delay requirement with the help of a DSR provided that the initial detection of available channels across the entire band is completed in a timely manner. Otherwise, as channel conditions vary, the CR cannot start operation until a new spectrum scan is completed and the availability of the channel is validated.

2.1. Prior Efforts. The research around sensing in cognitive radio has been extensive. There are several well researched techniques.

(1) Blind Sensing Algorithms. The technique is based on oversampling the received signal or by employing multiple receives antennas. The algorithm does not require knowledge of the channel or of the noise power (i.e., blind). When the primary signal is present, the signal statistics computed will differ much more in value from each other, than when the primary signal is not present $[13,14]$.

(2) Cooperative Sensing. It defines two protocols:

(i) Noncooperative (NC) Protocol. All users detect the primary user independently. However the first user to detect the presence of the primary user informs the other users through the central controller (distributed sensing).

(ii) Totally Cooperative (TC) Protocol. Two users operating in the same carrier, if placed sufficiently near each other, cooperate to find the presence of the primary user. The first user to detect the presence of the primary user informs the others through the central controller. 
(iii) Agility is measured as the probability of detection of noncooperative divided by probability of detection of cooperative protocol. The paper estimates that maximum gain in using the technique is $11 \%[15$, 16].

(3) PU LO Leakage Detection. Technique is based on the possibility of detecting primary receivers by exploiting the local oscillator (LO) leakage power emitted by the RF front end of primary receivers [17].

(4) Radio Identification-Based Sensing. A complete knowledge about the spectrum characteristics can be obtained by identifying the transmission technologies used by primary users.

(i) Several features are extracted from the received signal and they are used for selecting the most probable primary user technology by employing various classification methods. Features obtained by energy detector-based methods are used for classification. Channel bandwidth and its shape are used in reference features. Channel bandwidth is found to be the most discriminating parameter among others [18].

(5) Cyclostationary Feature Detection. To improve spectrum sensing sensitivity, cyclostationary feature detection computes the autocorrelation of received signal before the spectral correlation detection. The technique is based on the fact that modulated signals are in general coupled with sine wave carriers, pulse trains, or cyclic prefixes which result in built-in periodicity. The periodicity helps extracting information about the received signal such as modulation, pulse shape, and bandwidth [19].

None of the approaches described above address the requirements for time sensitive applications. As a matter of fact, several of these techniques actually lengthen the time required to search for appropriate CR channels.

In Table 1, "detection time" is the time required to scan the entire bandwidth, "detection ability" is the ability to correctly predict the presence or absence of a signal, "complexity" refers to the implementation complexity, "dependency" is the need for the sensing receiver to depend on another user, a base station or a master controller to perform sensing, and finally the "overall performance" is summarized in the last column. It is clear that none of the previous work actually addresses the timely sensing requirement of CR. The dedicated sensing receiver (DSR) architecture is presented in the next subsection.

2.2. The Dedicated Sensing Receiver. Based on the implementation and operational challenges described above, our proposed approach is to separate the continuous sensing function from the main CR receiver. The Dedicated Sensing Receiver (DSR) addresses several of the issues discussed earlier. The block diagram of the proposed architecture is shown in Figure 1.

At the heart of the DSR is a learning algorithm that continuously scans the spectrum and prioritizes the available channels in a look up table (LUT). In order to speed up sensing, both the main receiver and the DSR in Figure 1 perform the coarse sensing in essence sharing the work between the two receivers. Once the initial results in the LUT, the DSR performs the fine sensing on the candidate channels. In order to avoid conflict with a PU or another secondary user, continuous channel monitoring is done via detectors in the analog domain because of their fast response time. In order to take full advantage of the DSR, a radio architecture and especially the phase locked loop must be able to quickly hop and settle onto the desired frequency. Without an agile PLL, the system scan time would be gated by the radio hardware. The overall PLL design is critical to the performance, cost and complexity of the CR specifically across wideband operation. One important aspect of the cognitive radio network is to insure that the CR does not interfere with a PU or another SU in the band. In our implementation in [8], we proposed the analog RF detector shown as the "RF Coarse Sensing" in Figure 1 to monitor and suspend transmission if a detected signal surpasses a preset threshold.

2.3. Scan Time Calculations. Throughout the paper, we use the subscript "crs" to denote parameters associated with coarse sensing while "fin" is used for fine sensing. The overall system bandwidth of interest $B_{\text {sys }}$ is divided into $\beta$ coarse bins (each bin with bandwidth $B_{\text {crs }}$ ). Each coarse bin is further divided into $\alpha$ fine bins (with fine bin bandwidth corresponding to $B_{\text {fin }}$ ) as shown in Figure 2.

From Figure 2, it is clear that

$$
B_{\text {crs }}=\alpha B_{\text {fin }}, \quad \text { where } \alpha=1,2,3,4, \ldots
$$

In practical implementations, FFTs have widely used the split-radix FFT algorithm [20]. The number of real additions and multiplications needed for a $2^{N}$ points FFT (with $N$ $>1$ ) is given by $4 N \log _{2} N-6 N+8$. The resolution of the estimation is proportional to $N$. Hence, the resolution increases as $N$ increases. For fine sensing,

$$
B_{\text {fin }}=N F_{\text {res }} \text {, }
$$

where $F_{\text {res }}$ is the resolution of the sensing. The total time to perform a discrete Fourier Transform (DFT) is given by

$$
T_{\mathrm{DFT}}=\frac{1}{F_{\mathrm{DSP}}}\left(4 N \log _{2} N-6 N+8\right),
$$

where $F_{\mathrm{DSP}}$ is the DSP operating frequency. For simplicity, assume that the DSP is capable of performing one addition and one multiplication per clock cycle, the total sensing time for coarse and fine sensing of the total bandwidth is given by

$$
T_{\mathrm{crs}}=\frac{B_{\mathrm{SYS}}}{B_{\mathrm{crs}}} T_{\mathrm{DFT}} \text {. }
$$

As shown in Figure 1, the CR has two receiver chains: the main receiver and the DSR. With two available receivers, one would share the load across the two receivers. One can also expand this concept from 2 receivers to $M$ receivers operating 
TABLE 1: Prior work summary.

\begin{tabular}{|c|c|c|c|c|c|}
\hline & Detection time & Detection ability & Complexity & Dependencies & $\begin{array}{c}\text { Overall } \\
\text { performance }\end{array}$ \\
\hline $\begin{array}{l}\text { Base sensing } \\
\text { receiver }\end{array}$ & Too slow & $\begin{array}{l}\text { OK in } \\
\text { narrowband apps }\end{array}$ & $\bigcirc$ & 0 & $\begin{array}{l}\text { Solution } \\
\text { workable in low } \\
\text { bandwidth } \\
\text { solutions }\end{array}$ \\
\hline Blind sensing & $\begin{array}{l}\text { Fear of false } \\
\text { positive }\end{array}$ & $\begin{array}{l}\text { Because of } \\
\text { "comparative } \\
\text { sensing" might } \\
\text { miss low SNR } \\
\text { solutions }\end{array}$ & $\bigcirc$ & $\bigcirc$ & $\begin{array}{l}\text { Fear of missing } \\
\text { available channels } \\
\text { or false positives. }\end{array}$ \\
\hline $\begin{array}{l}\text { Cooperative } \\
\text { sensing- } \\
\text { distributed }\end{array}$ & $\begin{array}{l}\text { Each user must } \\
\text { still scan and detect } \\
\text { the band }\end{array}$ & $\begin{array}{l}\text { Sharing helps } \\
\text { improve detection }\end{array}$ & $\bigcirc$ & $\begin{array}{l}\text { Requires the } \\
\text { cooperation of } \\
\text { others in the } \\
\text { network }\end{array}$ & $\begin{array}{l}\text { Too slow and } \\
\text { needs input of } \\
\text { others }\end{array}$ \\
\hline $\begin{array}{l}\text { Cooperative } \\
\text { sensing- } \\
\text { centralized }\end{array}$ & $\begin{array}{l}\text { Time may be } \\
\text { accelerated with } \\
\text { help from BS }\end{array}$ & $\begin{array}{l}\text { Sharing helps } \\
\text { improve detection }\end{array}$ & $\bigcirc$ & $\begin{array}{l}\text { Requires the } \\
\text { cooperation of } \\
\text { others in the } \\
\text { network }\end{array}$ & $\begin{array}{l}\text { Improved time, } \\
\text { but requires } \\
\text { infrastructure and } \\
\text { may be limited in } \\
\text { frequency } \\
\text { operations. }\end{array}$ \\
\hline $\begin{array}{l}\text { Cooperative } \\
\text { sensing-Totally } \\
\text { cooperative }\end{array}$ & $\begin{array}{c}\text { Time is gated by } 2 \\
\text { or more CR sensing } \\
\text { the same channel }\end{array}$ & $\begin{array}{l}\text { Sharing helps } \\
\text { improve detection }\end{array}$ & $\bigcirc$ & $\begin{array}{l}\text { Requires the } \\
\text { cooperation of } \\
\text { others in the } \\
\text { network }\end{array}$ & $\begin{array}{l}\text { Improved time, } \\
\text { but requires } \\
\text { infrastructure and } \\
\text { may be limited in } \\
\text { frequency } \\
\text { operations. }\end{array}$ \\
\hline $\begin{array}{l}\text { PU LO leakage } \\
\text { detection }\end{array}$ & $\begin{array}{l}\text { Limited to the PU } \\
\text { bands }\end{array}$ & $\begin{array}{l}\text { Solution very } \\
\text { limited to a known } \\
\text { band }\end{array}$ & $\bigcirc$ & $\begin{array}{l}\text { Need prior } \\
\text { knowledge of PU }\end{array}$ & $\begin{array}{l}\text { Very limited } \\
\text { solution }\end{array}$ \\
\hline $\begin{array}{l}\text { Radio } \\
\text { identification } \\
\text { based sensing }\end{array}$ & $\begin{array}{l}\text { Limited to the PU } \\
\text { bands }\end{array}$ & $\begin{array}{l}\text { Solution very } \\
\text { limited to a known } \\
\text { band }\end{array}$ & 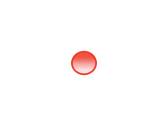 & $\begin{array}{l}\text { Need prior } \\
\text { knowledge of PU }\end{array}$ & $\begin{array}{l}\text { Very limited } \\
\text { solution }\end{array}$ \\
\hline Cyclostationary & $\begin{array}{l}\text { Detection time } \\
\text { slows down } \\
\text { considerably }\end{array}$ & 0 & 0 & 0 & $\begin{array}{l}\text { Better detection } \\
\text { ability but much } \\
\text { worse time }\end{array}$ \\
\hline $\begin{array}{l}\text { Network with } \\
\text { beacon }\end{array}$ & $\begin{array}{l}\text { Leverages beacon } \\
\text { to detect signal, but } \\
\text { limited to beacon } \\
\text { freq. bands }\end{array}$ & $\begin{array}{l}\text { Solution very } \\
\text { limited to a known } \\
\text { band }\end{array}$ & 0 & $\begin{array}{c}\text { Requires } \\
\text { cooperation from } \\
\text { beacon }\end{array}$ & $\begin{array}{l}\text { Very limited } \\
\text { application can } \\
\text { help avoid } \\
\text { interference }\end{array}$ \\
\hline
\end{tabular}

Best solution $\bigcirc$ Good $\bigcirc$ Adequate $\bigcirc$ Inadequate $\bigcirc$ Unworkable.

in parallel to reduce the scan time, where each receiver is tasked to scan a $1^{\prime} / M$ of the desired frequency band.

Combining (1), (2), and (3) and assuming that in coarse mode, $M$ receivers share the sensing load, we can write $T_{\text {crs }}$ and $T_{\text {fin }}$ as:

$$
\begin{aligned}
& T_{\text {crs }}=\frac{B_{\text {SYS }}}{\alpha M N_{\text {crs }} F_{\text {res }} F_{\mathrm{DSP}}}\left[4 N_{\text {crs }} \log _{2}\left(N_{\text {crs }}\right)-6 N_{\text {crs }}+8\right], \\
& T_{\text {fin }}=\frac{\alpha}{F_{\mathrm{DSP}}}\left[4 N_{\text {fin }} \log _{2}\left(N_{\text {fin }}\right)-6 N_{\text {fin }}+8\right],
\end{aligned}
$$

where $N_{\text {crs }}$ and $N_{\text {fin }}$ are the number of FFT points used in coarse and fine mode, respectively.

In order to compute the overall system sensing time we need to include the radio tuning time which is mostly dominated by the PLL lock times. Let us define three different
PLL locks times: $T_{\text {init }}$ which is the initial lock time, $T_{\text {PLL_crs }}$ which is PLL lock time for a coarse step, and $T_{\text {PLL_fin }}$ which is PLL lock time for a fine step. Hence, the total PLL sweep time $T_{\text {PLL_crs }}$ during the sensing operation is give by

$$
T_{\text {PLL_SYS }}=T_{\text {init }}+\alpha \beta T_{\text {PLL_fin }}+\beta T_{\text {PLL_crs }_{\text {_rs }}} .
$$

After the coarse scan, only a fraction of the channels is expected to be fine scanned. We define a term $\rho$ whereas $\rho$ is defined as the percentage of coarse bins that are identified as candidate channels after coarse sensing. In other words, if the entire band was available then $\rho=1$ which means that every coarse bin must be submitted for fine sensing. Conversely, $\rho=0$ means that the coarse sweep identified that all bins 


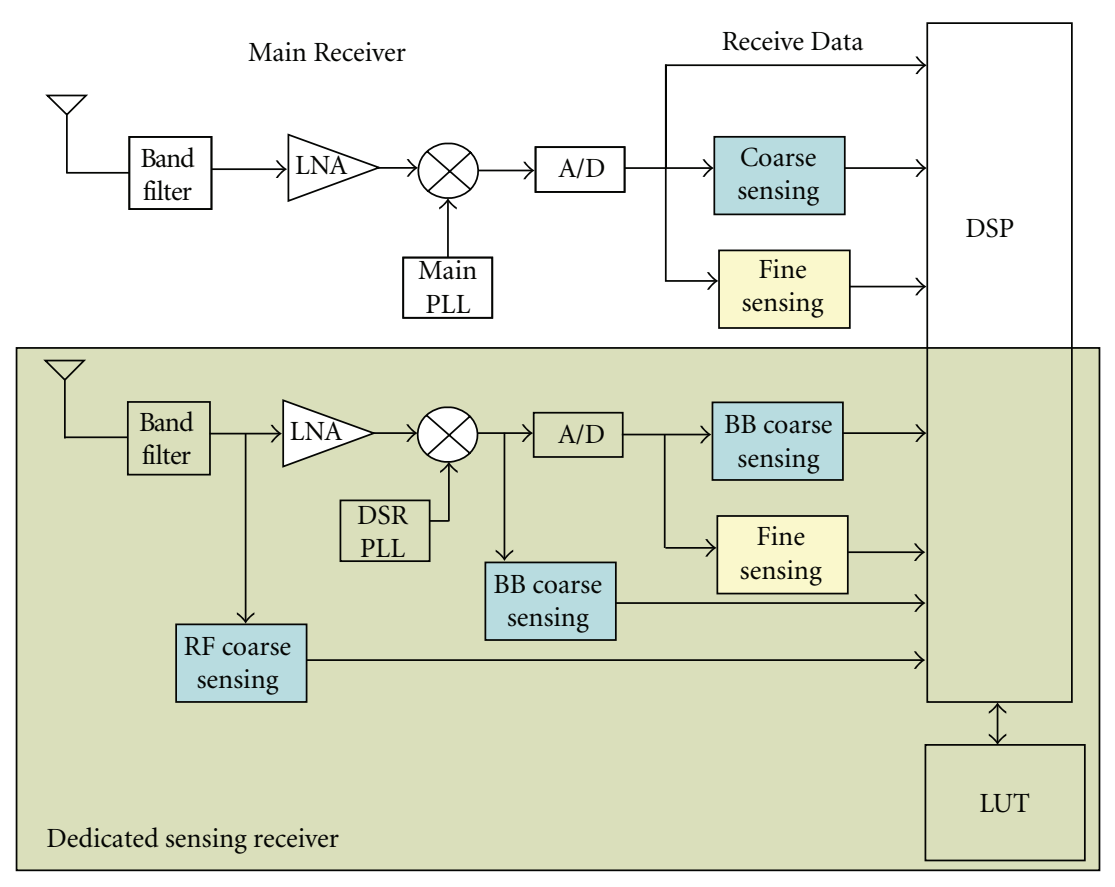

Figure 1: Proposed block diagram.

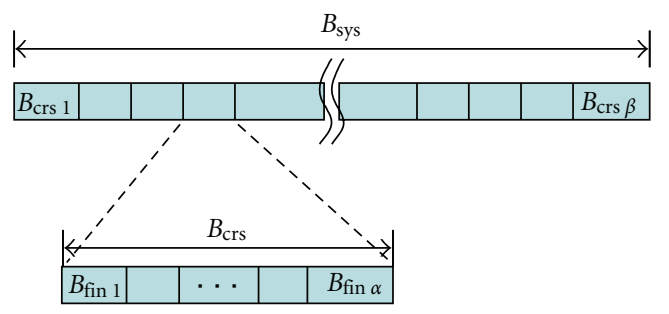

$B_{\mathrm{sys}}=\beta \cdot B_{\mathrm{crs}} \quad$ system $\mathrm{BW}$ is divided into $\beta$ coarse bins

$B_{\mathrm{crs}}=\alpha \cdot B_{\mathrm{fin}} \quad$ each coarse bin is divided into $\alpha$ fine bins

FIgURE 2: Channel model.

are occupied and hence no need for fine sensing. The overall system scan time is defined as

$$
\begin{aligned}
T_{\mathrm{SYS}}= & \frac{B_{\text {SYS }}}{\alpha M N_{\text {crs }} F_{\text {res }} F_{\mathrm{DSP}}}\left[4 N_{\text {crs }} \log _{2}\left(N_{\text {crs }}\right)-6 N_{\text {crs }}+8\right] \\
& +\frac{\alpha \beta \rho}{F_{\mathrm{DSP}} \cdot M}\left[4 N_{\text {fin }} \log _{2}\left(N_{\text {fin }}\right)-6 N_{\text {fin }}+8\right] \\
& +T_{\text {init }}+\frac{\alpha \beta \rho}{M} T_{\text {PLL_fin }}+\frac{\beta}{M} T_{\text {PLL_crs. }}
\end{aligned}
$$

Equation (7) corresponds to the overall system scan time for the proposed DSR. However, this equation assumes perfect detection and no false alarm during coarse scanning. In order to characterize the sensing time accurately, the probability of detection and false alarm rate of coarse scanning must be incorporated into (7).
2.4. Detection and False Alarm Probability. For the purposes of this paper, we assume that energy detection is used for detecting channel availability. The received signal is filtered then passed through a square law detector and integrated over a sensing time. We define (1) $P_{d}$ as the detection probability; (2) $P_{\mathrm{fa}}$ as the false alarm probability; (3) $D_{t}$ as the threshold level for the detection rule; (4) $J$ as the implementation penalty metric that models the additional wasted time needed to recover from a false alarm and resume the search process; $M$ as the number of receivers. In the case of the DSR shown in Figure 1, $M=2$. We further define $L$ as the actual number of idle coarse channels and $K$ as the actual number of idle fine channels. Hence, $\rho$ can be represented in term of $L$ as

$$
\rho=\frac{L}{\beta} .
$$

Assuming a serial search is performed, the mean detection time $\bar{T}_{\text {det }}$ corresponds to [21]

$$
\bar{T}_{\mathrm{det}}=\bar{S}_{\mathrm{det}}\left(T_{s}+T_{i}\right),
$$

where,

$$
\bar{S}_{\mathrm{det}}=\frac{(\beta-L) J P_{\mathrm{fa}}+\beta}{P_{d}(L+1)} .
$$

$\bar{S}_{\text {det }}$ is the average number of steps in channel scanning prior to a success (i.e., detecting an idle channel). $T_{s}$ is the switching time. However, since we have 2 different switching times in this system $T_{\text {PLL_crs }}$ and $T_{\text {PLL_fin }}$, we set $T_{s}=T_{\text {PLL_crs }}$ when we generate a coarse detection time. Similarly, we set $T_{s}=T_{\text {PLLfin }}$ in order to determine the fine detection time. $T_{i}$ is the integration time required for making a decision. 
From (9) and (10), we can write down the mean detection time in coarse mode

$$
\begin{aligned}
\bar{T}_{\text {det_crs }}= & \frac{(\beta-L) J P_{\mathrm{fa}}+\beta}{P_{d}(L+1)} \\
& \times\left[T_{\text {PLL_crs }}+\frac{1}{F_{\text {DSP }}}\left(4 N_{\text {crs }} \log _{2} N_{\text {crs }}-6 N_{\text {crs }}+8\right)+T_{\text {init }}\right]
\end{aligned}
$$

Similarly, $\bar{T}_{\text {det }}$ for the fine mode can be derived and plugged into (7) in order to determine the overall mean time detection of system defined by $\bar{T}_{\text {sys }}$ in

$$
\begin{aligned}
\bar{T}_{\text {sys }}= & \left(\frac{B_{\text {sys }}}{\alpha M N_{\text {crs }} F_{\text {res }}}+\frac{(\beta-L) J \cdot P_{\mathrm{fa}}+\beta}{P_{d}(L+1)}\right) A_{\mathrm{crs}} \\
& +\left(\frac{\alpha \beta \rho}{M F_{\mathrm{DSP}}}+\frac{(\alpha-K) J \cdot P_{\mathrm{fa}}+\alpha}{P_{d}(K+1)}\right) A_{\mathrm{fin}}+T_{\mathrm{init}} \\
& +\left(\frac{\alpha \beta \rho}{M}+\frac{(\alpha-K) J \cdot P_{\mathrm{fa}}+\alpha}{P_{d}(K+1)}\right) T_{\mathrm{PLL}_{-} \mathrm{fin}} \\
& +\left(\frac{\beta}{M}+\frac{(\beta-L) J \cdot P_{\mathrm{fa}}+\beta}{P_{d}(L+1)}\right) T_{\mathrm{PLL}_{\mathrm{f}} \mathrm{crs}},
\end{aligned}
$$

where

$$
\begin{aligned}
& A_{\mathrm{crs}}=4 \cdot N_{\mathrm{crs}} \log _{2} N_{\mathrm{crs}}-6 N_{\mathrm{crs}}+8 \\
& A_{\mathrm{fin}}=4 \cdot N_{\mathrm{fin}} \log _{2} N_{\mathrm{fin}}-6 N_{\mathrm{fin}}+8 .
\end{aligned}
$$

As expected, there are several parameters that affect the overall mean time detection of a two-stage sensing system as proposed in Section 2.1. Equation (12) illustrates that sensing time is influenced by environmental parameters such as $B_{\text {sys }}, \mathrm{SNR}, K$, and $L$, while other factors affecting $\bar{T}_{\text {sys }}$ are under user control such as number of FFT points, $\alpha$ and $\beta$. In the next section, we work to minimize $\bar{T}_{\text {sys }}$ by appropriately choosing user defined parameters. Although the detection of the signal is critical, this work focuses on the ability of the system to quickly and efficiently scan and track available channels. Nevertheless, the detection and false alarm probabilities are integrated into the total mean detection time of the system and hence are used to set a threshold for detection. $P_{d}$ and $P_{\mathrm{fa}}$ are assumed to be given based on the detector performance and the received signal quality.

\section{System Optimization}

The main goal of the sensing receiver is to detect available channels quickly and reliably. Most importantly, it is critical to reduce the number of false alarms. In examining (12) above, there are some very obvious ways to minimize $\bar{T}_{\text {sys }}$ such as reducing initialization time $\left(T_{\text {init }}\right)$ and the PLL lock times ( $T_{\text {PLL_crs }}$ and $T_{\text {PLL_fin }}$ ). However, the PLL lock time has physical implementation limitations [8]. The critical PLL parameters that affect the receiver performance (besides center frequency and power consumption) are switching time, phase noise, and spurs (also called reference sideband).
While the phase noise and spurs are directly proportional to the loop bandwidth, the switching time is inversely proportional [22]. In other words, as the loop bandwidth increases to accommodate faster lock time, the PLL phase noise and sideband spurs degrade which in turn cause the sensitivity of the receiver to degrade. Hence, the PLL lock time implementation is restricted by the phase noise budget within the radio design.

Another method used to reduce sensing time is to make appropriate choices for coarse and fine bins, that is, selecting optimal $\beta$ and $\alpha$ to minimize the overall mean detection time. In order to minimize $\bar{T}_{\text {sys }}$, we can employ the standard strategy of equating the partial derivatives of (12) with respect to $\beta$ and $\alpha$ to zero. However, $P_{d}$ and $P_{\mathrm{fa}}$ complicate this computation since they exhibit a dependence on the sensing or detection bandwidth which is directly proportional to $\beta$ and $\alpha$. Therefore, we first simplify (12) by approximating $P_{d}$ and $P_{\mathrm{fa}}$.

Assuming noncoherent square-law detection is used, $P_{d}$ and $P_{\mathrm{fa}}$ corresponds to [21]

$$
\begin{aligned}
& P_{d} \approx Q\left(\frac{D_{t}-2 T_{\text {sense }} B_{\text {sense }}(1+\mathrm{SNR})}{2 \sqrt{T_{\text {sense }} B_{\text {sense }}} \sqrt{1+2 \mathrm{SNR}}}\right), \\
& P_{\mathrm{fa}} \approx Q\left(\frac{D_{t}-2 T_{\text {sense }} B_{\text {sense }}}{2 \sqrt{T_{\text {sense }} B_{\text {sense }}}}\right),
\end{aligned}
$$

where $Q(x)=(1 / \sqrt{2 \pi}) \int_{x}^{\infty} e^{-\tau^{2} / 2} d \tau$.

As described earlier, $T_{\text {sense }}$ and $B_{\text {sense }}$ constitute the sensing time and the sensing bandwidth that is directly proportional to the bin bandwidth. Equation (14) can be approximated using a sigmoid function. The authors in [23] used a gradient approximation of the sigmoid function that was used for a fast algorithm for learning largescale preference relations. The relationship between the sigmoid function and complementary error function can be approximated as [23]

$$
\sigma(z)=\left(1+e^{-z}\right)^{-1} \approx 1-\frac{1}{2} \operatorname{erfc}\left(\frac{\sqrt{3} z}{\sqrt{2} \pi}\right)
$$

Recall that

$$
Q(z)=\frac{1}{2} \operatorname{erfc}\left(\frac{z}{\sqrt{2}}\right)
$$

By combining (15) and (16), Q(z) may be approximated as

$$
Q\left(z \frac{\sqrt{3}}{\pi}\right) \approx 1-\left(1+e^{-z}\right)^{-1}
$$

We can use the approximation in (17) to find a simplified expression for $P_{d}$ and $P_{\mathrm{fa}}$ in (14). After substituting these 
updated $P_{d}$ and $P_{\mathrm{fa}}$ expressions in (12), the simplified approximation of $\bar{T}_{\text {sys }}$ is shown in

$$
\begin{aligned}
\bar{T}_{\text {sys }}(\alpha, \beta)= & \left(\frac{A_{\text {crs }}}{M \cdot F_{\mathrm{DSP}}}+\frac{T_{\mathrm{PLL}_{\text {crrs }}}}{M}\right) \beta+\frac{A_{\text {crs }}+T_{\mathrm{PLL} \_ \text {crs }}}{L+1} \\
& \times\left[J e^{x(y-1)}(\beta-L)+\beta e^{x}\right] \\
& +\left(\frac{L A_{\mathrm{fin}}}{M F_{\mathrm{DSP}}}+\frac{L \cdot T_{\mathrm{PLL} \_ \text {fin }}}{M}\right) \alpha+\frac{A_{\text {fin }}+T_{\text {PLL_fin }}}{K+1} \\
& \times\left[J e^{v(y-1)}(\alpha-K)+\alpha e^{\nu}\right]+T_{\mathrm{init}},
\end{aligned}
$$

where

$$
\begin{aligned}
& y=\frac{\mathrm{SNR}+1}{\sqrt{1+2 \mathrm{SNR}}}, \\
& x=\sqrt{\frac{\pi^{2} A_{\mathrm{crs}} B_{\mathrm{sys}}}{6 \beta \cdot F_{\mathrm{DSP}}}}, \\
& v=\sqrt{\frac{\pi^{2} A_{\mathrm{fin}} B_{\mathrm{sys}}}{6 \alpha \beta \cdot F_{\mathrm{DSP}}}} .
\end{aligned}
$$

The expression in (18) characterizes the mean scan time as a function of a number of system parameters. Specifically, we can show that under certain conditions, $\bar{T}_{\text {sys }}$ in (18) is a convex function with respect to $\alpha, \beta$ as its Hessian is positive definite. The conditions for convexity are $\alpha>K$ (i.e., the number of idle fine channels is less than the total number of fine channels), $\beta>L$ (i.e., the number of idle coarse channels is less than the total number of coarse channels) and $y>1$ (i.e., the SNR is real). All three conditions for convexity are practical and essential. Since $\bar{T}_{\text {sys }}$ is convex in $\alpha$ and $\beta$ (see in the appendix), we can determine the optimal choice for the number of coarse and fine bins (that minimize minimum scan time) as the values that force the derivative of (18) to 0 .

The partial derivate of (18) with respect to $\beta$ corresponds to

$$
\begin{aligned}
\frac{\partial}{\partial \beta} \bar{T}_{\text {sys }}= & \frac{A_{\text {crs }}}{M F_{\mathrm{DSP}}}+\frac{T_{\mathrm{PLL} \_ \text {crs }}}{M}+\frac{A_{\text {crs }}+T_{\mathrm{PLL} \_ \text {crs }}}{L+1} \\
& \times\left[J e^{x(y-1)} 1-\frac{1}{2}(y-1) x\right. \\
& \left.\quad+\frac{J L}{2 \sqrt{\beta}}(y-1) x e^{x(y-1)}+\left(1-\frac{1}{2} x\right) e^{x}\right] .
\end{aligned}
$$

Similarly, we can write down the partial derivative of $\bar{T}_{\text {sys }}$ with respect to $\alpha$ :

$$
\begin{aligned}
\frac{\partial}{\partial \alpha} \bar{T}_{\text {sys }}= & \frac{L A_{\text {fin }}}{M F_{\mathrm{DSP}}}+\frac{L T_{\text {PLL_crs }}}{M}+\frac{A_{\text {fin }}+T_{\text {PLL_fin }}}{K+1} \\
\times & {\left[J e^{v(y-1)} 1-\frac{1}{2}(y-1) v\right.} \\
& \left.\quad+\frac{J K}{2 \sqrt{\alpha}}(y-1) v e^{v(y-1)}+\left(1-\frac{1}{2} v\right) e^{v}\right] .
\end{aligned}
$$

As expected, (21) depends on both $\beta$ and $\alpha$ while (20) is only dependent on $\beta$. That is, as the sensing receiver initiates a coarse search, the number of coarse bins is not dependent on the fine scan. However, once the coarse scan is completed, the fine scan is dependent on the results of the coarse scan (i.e., dependent on $\beta$ ). The fine scan is initiated according to the priority set in the LUT set after the coarse scan is completed. We can set (20) and (21) to zero and solve for $\beta$ and $\alpha$ that minimize $\bar{T}_{\text {sys }}$. We employ numerical nonlinear solvers in order to find the solution to (20) and (21). The results from the optimization and its physical interpretation are presented in the next section.

\section{Simulation Results}

In this paper, our goal is to find the optimal bin size for coarse and fine sensing under given channel conditions and design implementation of the radio. As the spectrum becomes more and more crowded, the number of idle channels for coarse $(\mathrm{K})$ and fine sensing $(L)$ decreases and hence, on average, it would take the sensing receiver a longer time to identify an appropriate channel for CR operation (i.e., increases). Similarly, the physical implementation is mostly defined by the user given restrictions on cost, power, performance, and so forth. For example, the total time to perform a DFT in (3) is inversely proportional to the speed of operation of the DSP. A brute force approach would be for the designer to choose the fastest DSP available. However, fast DSP comes with a premium in cost and power consumption that may or may not necessarily affect the overall system performance. The solution to this problem is fine balance between coarse and fine sensing.

In this section, the simulation results are presented in two parts. In the first part, we focus on minimizing $\bar{T}_{\text {sys }}$ in (12). We provide a better insight on the dependence of minimum $\bar{T}_{\text {sys }}$ with respect to input variables such as the number of FFT points, DSP operating frequency, number of available channels (or spectrum crowding), assuming that $\beta$, $\alpha, P_{d}$, and $P_{\mathrm{fa}}$ are given. In the second part, we concentrate on optimizing $\beta$ and $\alpha$ in order to minimize $\bar{T}_{\text {sys }}$ given the channel conditions (e.g., $L, K$, and SNR) and the physical implementation of the radio (such as PLL initialization, PLL lock times, number of FFT points, and the DSP frequency $\left.F_{\mathrm{DSP}}\right)$.

4.1. Total Mean Detection Time $\bar{T}_{s y s}$. We simulate the total sensing time with respect to channel conditions and our choice of $\beta, \alpha$. The basic parameters for our environment are $J=2, N_{\text {crs }}=64, N_{\text {fin }}=512, M=2, F_{\mathrm{DSP}}=50 \mathrm{MHz}$, $B_{\text {sys }}=10 \mathrm{GHz}$ (broadband), $T_{\text {PLL_crs }}=10 \mathrm{~ms}, T_{\text {PLL_crs }}=$ 1 milliseconds and $T_{\text {inits }}=100$ milliseconds. In the first simulation, we want to better understand how $\bar{T}_{\text {sys }}$ is affected by the increase of the number of users. As the number of users increases, the occupancy of the spectrum increases and hence the number of idle channels suitable for CR operation decreases. Recall that $\rho$ constitutes the fraction of available coarse channels that are scanned in fine mode. On one hand, we want $\rho$ to be as small as possible in order to minimize fine scan. However, if $\rho$ is too small, then the occupancy of 


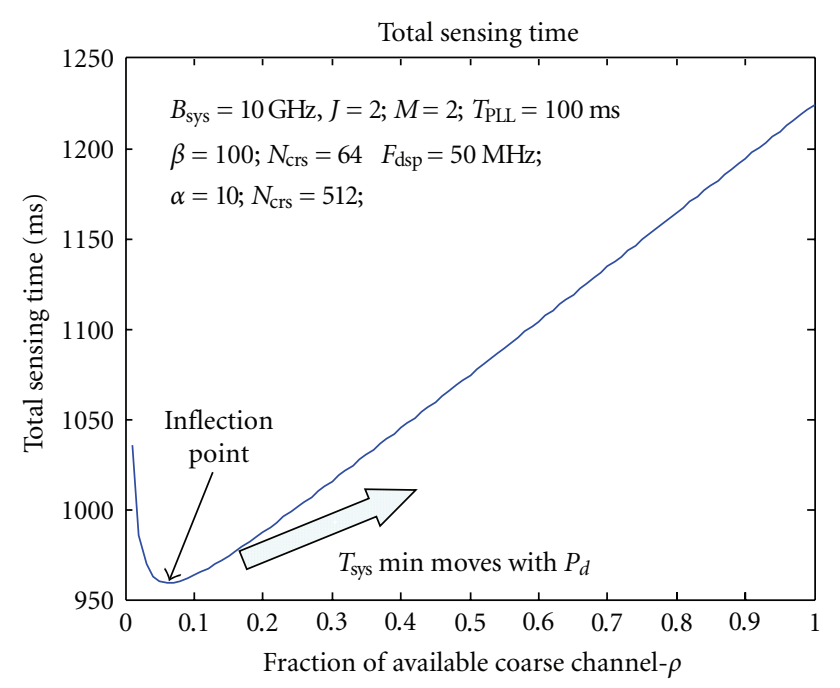

Figure 3: Total mean sensing time versus fraction of available coarse channels.

the channel is high and therefore it takes the DSR longer to find an available channel. The simulation of $\rho$ versus $\bar{T}_{\text {sys }}$ is shown in Figure 3. As expected, as the number of available candidate channel $(\rho)$ increases, the algorithm identifies and tags additional channels for fine sensing. Therefore the lower the number of candidate channels needed to be fine scanned the lower the total sensing time. However, as the number of candidate channels $(\rho)$ decreases to value typically $<10 \%$, the total sensing time reverses course and begins to increase. At low $\rho$ values, it is less probable that the sensing receiver finds an available channel quickly. Hence, the total sensing time increases due to the lack of available channels that are viable for CR operation. The dependence in Figure 3 indicates an optimal $\rho$ value for minimizing sensing time. However, $\rho$ is a system parameter that is outside the control of the designer.

The minimum sensing location is dependent on the value of $P_{d}$. As $P_{d}$ increases, the $\bar{T}_{\text {sys }}$ minimum location increases while shifting to the right. The main reason for this shift is that as the probability of detection increases, the false alarm probability tends to increase. With an increasing number of misdetection, the total system mean time is affected by the factor $J$ which is an implementation penalty metric that models the additional wasted time needed to recover from a false alarm and resume the search process.

The question remains how would $\bar{T}_{\text {sys }}$ be affected by our choice of $\beta$ and $\alpha$ ? Since the coarse sensing time is much lower than the fine sensing time, $\bar{T}_{\text {sys }}$ is reduced if more of the detection is done in coarse mode. On the flip side, the resolution in coarse mode is lower than in fine mode and false alarms or false positive reading of the spectrum would cause the DSR to reset and resume the scanning process. This penalty is captured by parameter $J$ and the $F_{\text {res }}$ in (12). Using the same variables as defined above, we simulate the total mean sensing time versus $\beta$ and $\alpha$ is shown in Figure 4 with $\rho=.5$.

The relationship among $\beta, \alpha$, and $\bar{T}_{\text {sys }}$ is shown in Figure 4 . We can observe that the sensing time is typically

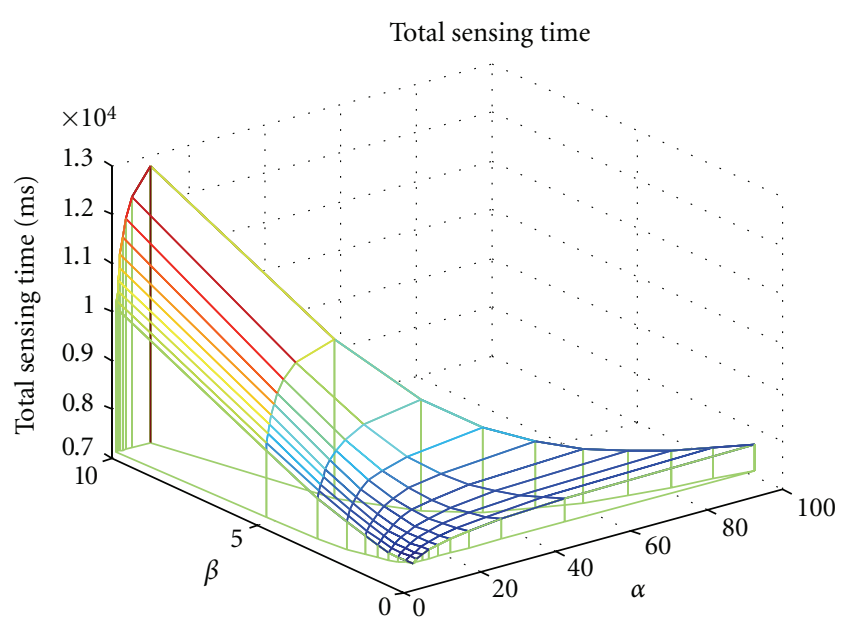

Figure 4: Total mean sensing time versus $\beta$ and $\alpha$.

lower at lower $\beta$ and as expected, increases as $\alpha$ increases. The resolution and the switching time in coarse mode start to have a much greater effect on $\bar{T}_{\text {sys }}$ than the computation of the N-point FFT. Given channel conditions and circuit implementation (on the PLL, e.g.), we expect to find a combination of $\beta, \alpha$ such that the total mean detection time is minimized. One would hope that the combination would give a global minimum and hence provide an optimal solution for the system. In the next subsection, we calculate $\beta$ and $\alpha$ such that $\bar{T}_{\text {sys }}$ is minimized.

4.2. Optimal $\beta$ and $\alpha$ for Minimum $\bar{T}_{\text {sys. }}$. With the detection time highly dependent on the coarse and fine bandwidths, we seek to find an optimal solution. This is a largescale unconstrained optimization with primarily two sets of variables (1) channel dictated variables such as SNR and implementation variables such as PLL lock times and (2) the choice of DSP. In this section, we study the effect of the aforementioned variables on the minimum mean detection time of the system. First, we simulate the effect of the given variables on optimal $\beta$ and $\alpha$. We seek to find the parameters in support of our algorithm such as number of FFT points in coarse and fine mode and bin sizes. Second, we present our results in a summary table format.

We use (20) and (21) to determine optimal $\alpha$ with respect to the channel variables (such as $K, \mathrm{SNR}$ ). The results are documented in Figures 5 and 6 . In Figure 5, we plot the effect of the number of available fine channels $K$ (or channel crowding) versus the optimal $\alpha$. As the channels become crowded (i.e., $K$ decreases), the probability of finding an idle channel decreases which requires additional sensing time. This phenomenon can be observed in Figure 5, where $K$ becomes a dominant factor as the number of idle channel decreases. Under the conditions shown in the figure, the effect of $K$ becomes less dominant when the number of fine available bins reaches $\sim 700$. The slope decreases by almost a factor of 5 between $K=100$ and $K=700$.

In Figure 6, we plot the effect of SNR on choice of $\alpha$. We note that as SNR increases, the number of required 


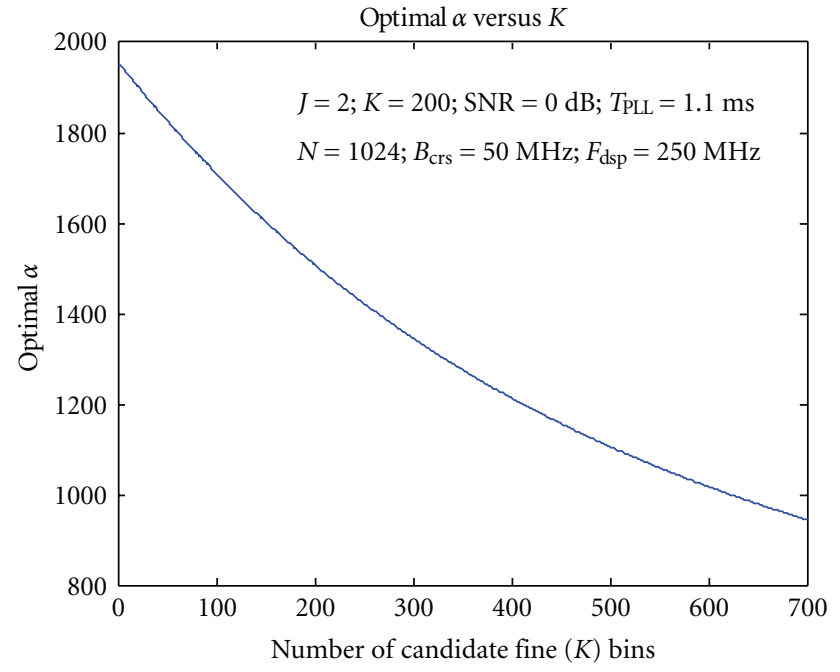

Figure 5: Optimal $\alpha$ versus number of available fine bins.

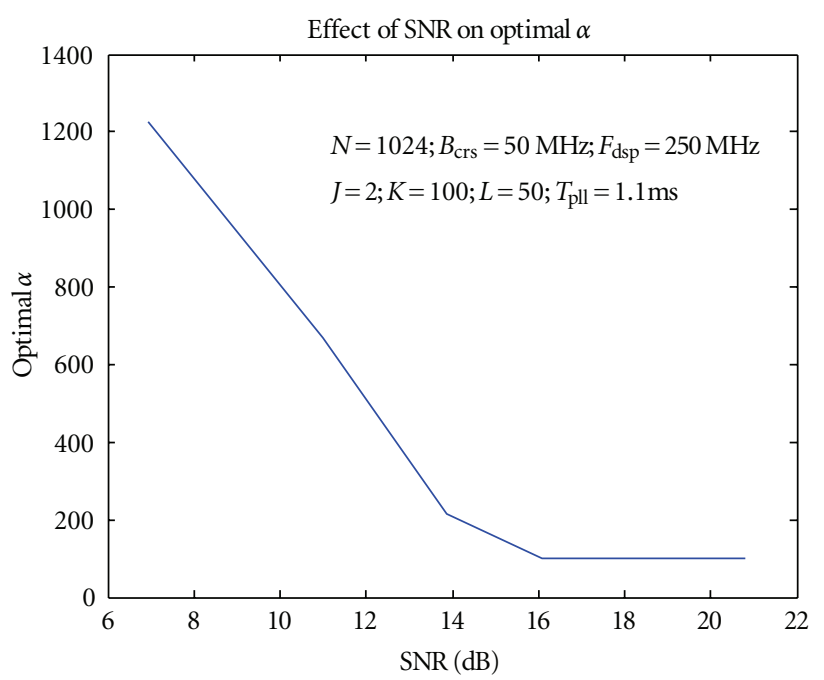

FIGURE 6: Optimal $\alpha$ versus SNR of received signal.

fine sensing bins decreases until it reaches the limit of our convexity condition $\alpha=K$, which basically states that all bins are available and may be used for CR operation. These results support our intuition that in order to minimize the overall scanning time, we need to perform less computation. Since the fine bins require more computation time, we seek to decrease the number of fine bins. That goal becomes more palatable at high SNR value where probability of detection is high and the probability of false alarm is low.

Similarly, we study the effect of the variables on the our choice of $\beta$. We document our results for $\beta$ in Figures 7 9. In Figure 7, we note that the number of available coarse bins $(L)$ affects $\beta$ in the same manner as $K$ affected $\alpha$. As the number of available bin increases, we expect a higher probability of detection and with a reduction in $\beta$, the overall $\bar{T}_{\text {sys }}$ decreases. In this example, we show that when there is only $10 \%$ available bins ( $K=50$ of a total of 500$)$, we need a large $\beta$ which basically states that the bandwidth

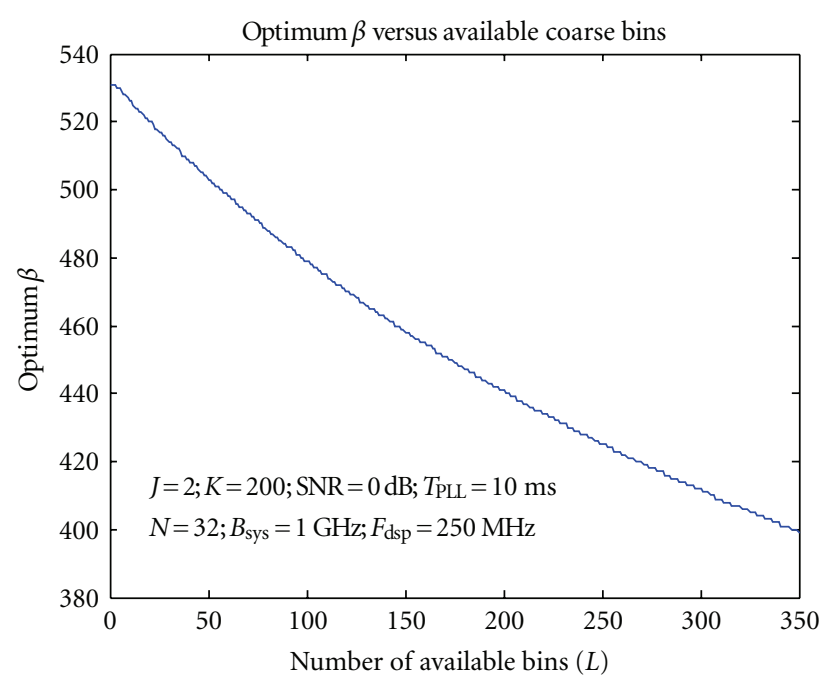

FIgURE 7: Optimal $\beta$ versus available coarse channels.

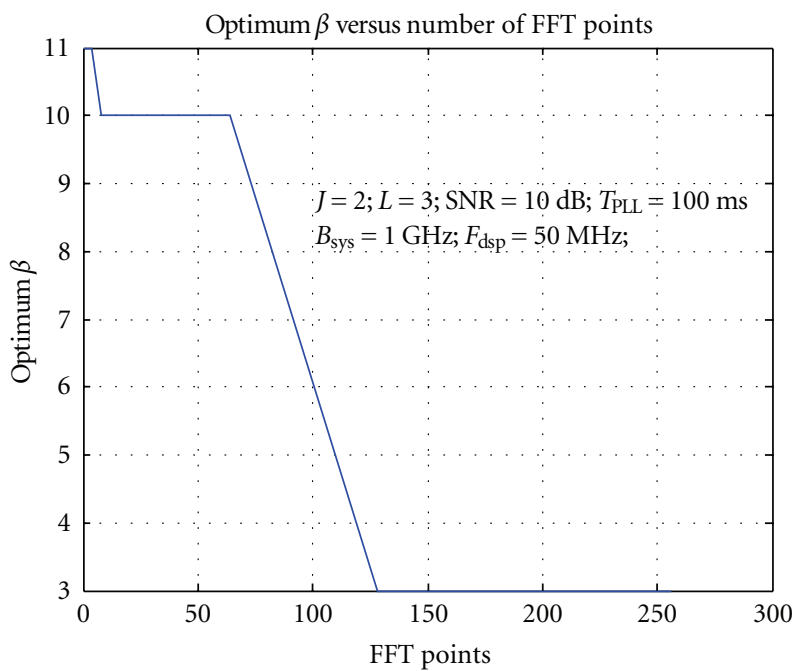

FIgURE 8: Optimal $\beta$ versus number of FFT points.

must be divided into small bands in order to find idle channels.

In Figure 8, we show the number of coarse N-point FFT calculation versus our choice of $\beta$. In this example, we set the fine scan mode FFT points to 512. In Figure 8, the number of bins decreases as the number of FFT points increases, until the limit condition for convexity of $\beta=L$ is reached. Another interpretation of the results is as the number of FFT points increases, it becomes less viable that a 2-stage scanning process is needed. One of the main advantages of going to a 2-stage sensing technique is to reduce the number of calculation by allowing a coarse mode to do a cursory search for available channels. As the number of coarse FFT points start to approach that of a fine sensing mode, the advantage and effectiveness of the coarse sensing mode is reduced.

Similar to Figure 6, the need for coarse bins decreases as the SNR increases. The results are captured in Figure 9. One 


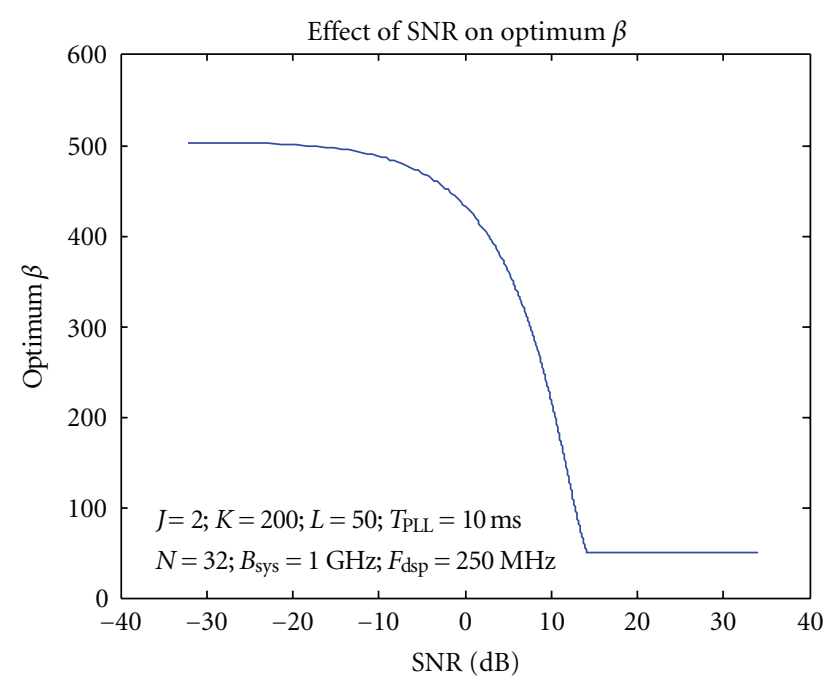

FIgURE 9: Effect of SNR on Choice of Optimal $\beta$.

TABLE 2: $\bar{T}_{\text {sys }}$ versus SNR.

\begin{tabular}{llcc}
\hline SNR & $\beta$ & $\alpha$ & $\bar{T}_{\text {sys }}(\mathrm{s})$ \\
\hline 15 & 43 & 998 & 1.57 \\
30 & 40 & 678 & 1.07 \\
60 & 39 & 589 & 0.938 \\
\hline
\end{tabular}

interesting aspect of the results that was not obvious with $\alpha$ is the fact that the required number of bins does not vary below a given SNR (in the example below $\sim-20 \mathrm{~dB}$ ). This result is the opposite effect of what we discussed earlier in Figure 8. As the SNR decreases, more and more bins are needed to a point where the coarse sensing bandwidth is small enough to start infringing on the need for fine sensing. When the SNR is high, the probability of detection increases, and therefore the need for additional coarse search bins is reduced until the limit condition of convexity $\beta=L$ is reached at which point $\beta$ can not be reduced further.

In order to better understand the sensitivity of our analysis on $\bar{T}_{\text {sys }}$, we show selected results below. The parameters used for these simulations are $T_{\text {PLL_crs }_{1}}=0.5 \mathrm{~ms}$, $T_{\mathrm{PLL}_{\text {ffin }}}=$ 0.1 mlliseconds, $M=2, J=2$, and $F_{\mathrm{DSP}}=250 \mathrm{MHz}$. In Table 1, we set $L=6, K=22, N_{\text {crs }}=64$, and $N_{\text {fin }}=2048$. Please note that by doubling SNR from 15 to 30 , the effect on $\alpha$ is a $32 \%$ reduction versus a $7 \%$ on $\beta$. This discrepancy in variation supports our earlier results. As SNR increases, the need for bins decreases. However, the sensing time is far greater for fine mode sensing than in coarse mode sensing. Hence, the algorithm gives the priority to reducing $\alpha$ over $\beta$ which has a greater affect on $\bar{T}_{\text {sys }}$. Recall that for time sensitive applications, the DSR surveys the desired band of operation, sorts and prioritizes the channels best suited for CR operation. After the channels are identified and stored, the DSR continuously monitors and reprioritize the channels as needed. In order to avoid storing "stale" data in the LUT, the overall $\bar{T}_{\text {sys }}$ must be minimized. Our goal is to optimize $\bar{T}_{\text {sys }}$ by minimizing the overall sensing time.
TABLE 3: $\bar{T}_{\text {sys }}$ versus $N_{\text {fin }}$.

\begin{tabular}{lccc}
\hline$N_{\text {fin }}$ & $\beta$ & $\alpha$ & $\bar{T}_{\text {sys }}(\mathrm{s})$ \\
\hline 1024 & 40 & 901 & 0.828 \\
2048 & 40 & 678 & 1.07 \\
4096 & 40 & 595 & 1.81 \\
\hline
\end{tabular}

TABle 4: $\bar{T}_{\text {sys }}$ versus Available Fine Channels $(K)$.

\begin{tabular}{llcc}
\hline$K$ & $\beta$ & $\alpha$ & $\bar{T}_{\text {sys }}(\mathrm{s})$ \\
\hline 22 & 40 & 678 & 1.07 \\
20 & 40 & 539 & 0.867 \\
10 & 40 & 115 & 0.275 \\
\hline
\end{tabular}

In Table 2, we set $L=6, K=22, N_{\text {crs }}=64$, and SNR $=30$. We vary the number of FFT points for the fine mode $\left(N_{\text {fin }}\right)$. As expected, by varying the $N_{\text {fin }}$, there is no impact on $\beta$ since it is independent of the coarse sensing, but there is a high impact on $\alpha$ and $\bar{T}_{\text {sys }}$.

As the number of FFT points increases, $\alpha$ decreases but the overall $\bar{T}_{\text {sys }}$ increases. Another parameter that has a high impact on $\bar{T}_{\text {sys }}$ is the activity in the spectrum. In our results, we showed that as the number of available channels decreases, we need additional bins (i.e., $\alpha$ increases) in order to identify idle channels. In Table 3, we set $L=6, N_{\text {fin }}=2048$, $N_{\text {crs }}=64$, and SNR $=30$ and we vary the $K$ variable. Please note that as $K$ increases, $\alpha$ decreases, and $\bar{T}_{\text {sys }}$ also decreases at a fast rate (Table 4 ).

In this example, the improvement on $\bar{T}_{\text {sys }}$ is drastic. Unfortunately, $K$ is a representation of the channel environment and it is not under user control.

\section{Conclusions}

In this paper, we propose the use of dedicated sensing receiver architecture with a 2-stage sensing algorithm required for time sensitive applications such as voice. We quantify the effect of channel variables (SNR, number of idle channels, etc.) and radio implementation parameters (PLL lock time, $\mathrm{N}$-point FFT, etc.) on the total mean detection time. We minimize our detection time by optimizing the coarse and fine bin sizes in our 2-stage sensing algorithm. In order to achieve an equilibrium point, we perform a large-scale optimization on the mean detection time with respect to bin sizes. Coarse sensing is faster than fine sensing, however, it is not as accurate. As the number of users in a channel increases, the number of fine bins increases which directly affects the total scan time. Hence, we optimize our sensing time by striking a balance between the fast, lower accuracy coarse detection versus the slower, more accurate fine sensing operation.

In our future work, we will focus on adaptively allocating the fine sensing bins with the coarse bins. In other words, we could have a different number of fine bins for each coarse bin. In the case of a busy spectrum, we would assign additional fine sensing bins, but this choice of bins in the busy spectrum band should not be perpetuated to other coarse bins when 
the activity is a lot lower. We believe that such efforts will further reduce the mean detection time.

\section{Appendix}

\section{A. Proof of Convexity}

Lemma 1. The sensing time as given in (18) is a convex function of $\beta$ and $\alpha$.

Proof. The sensing time is given as

$$
\begin{aligned}
\bar{T}_{\text {sys }}(\alpha, \beta)= & \left(\frac{A_{\text {crs }}}{M \cdot F_{\mathrm{DSP}}}+\frac{T_{\mathrm{PLL}_{\text {crrs }}}}{M}\right) \beta+\frac{A_{\text {crs }}+T_{\mathrm{PLL}_{\_} \text {rs }}}{L+1} \\
& \times\left[J e^{x(y-1)}(\beta-L)+\beta e^{x}\right] \\
& +\left(\frac{L A_{\text {fin }}}{M F_{\mathrm{DSP}}}+\frac{L \cdot T_{\mathrm{PLL} \_ \text {fin }}}{M}\right) \alpha+\frac{A_{\text {fin }}+T_{\mathrm{PLL} \_ \text {fin }}}{K+1} \\
& \times\left[J e^{v(y-1)}(\alpha-K)+\alpha e^{v}\right]+T_{\text {init. }} .
\end{aligned}
$$

In (A.1), the first and third terms are linear in $\alpha$ and $\beta$ and therefore convex. Let us take a closer look at the second term. The second term corresponds to the effect of sensing in coarse mode. It has two parts. Both parts are functions of $x$ and $y$ that are defined as

$$
\begin{aligned}
& x=\sqrt{\frac{\pi^{2} A_{\mathrm{crs}} B_{\mathrm{sys}}}{6 \beta \cdot F_{\mathrm{DSP}}}}, \\
& y=\frac{\mathrm{SNR}+1}{\sqrt{1+2 \mathrm{SNR}}} .
\end{aligned}
$$

From (A.3), $y$ is dependent on SNR and not on $\alpha$ and $\beta$, while $x$ is a function of $\beta$. Since $1 / \sqrt{p}$ is convex in $p, x$ is a convex function with respect to $\beta$. By invoking the properties (1) $e^{p}$ is convex if $p$ is convex, (2) the product of a convex function and a constant is convex, and (3) the product of two convex functions is convex if both functions are positive and nondecreasing, we have the following results.

(1) The product $e^{x(y-1)}(\beta-L)$ is convex as long as $\beta>L$.

(2) The product $\beta e^{x}$ is convex as long as $\beta>0$.

Since the sum of 2 convex functions multiplied by a constant is convex, the second term in (A.1) is convex. Similarly, we can show that the 4 th term which represents the fine sensing mode is also convex with respect to $\alpha$ as long as $\alpha>K$ and $\alpha>0$. Therefore, $\bar{T}_{\text {sys }}(\alpha, \beta)$ as defined in (A.1) is the sum of 4 convex terms and a constant and is therefore convex as long as (1) $\beta>L,(2) \alpha>K$, and (3) SNR is real. All three conditions of convexity are practical.

\section{References}

[1] XG Working Group, “The XG vision. Request for comments. Version 2.0,” Tech. Rep., BBN Technologies, 2005.
[2] DARPA WAND Industry Day, "XG Communications Program Overview," Feburary 2007.

[3] D. Cabric, S. M. Mishra, and R. W. Brodersen, "Implementation issues in spectrum sensing for cognitive radios," in Proceedings of the 38th Asilomar Conference on Signals, Systems and Computers, vol. 1, pp. 772-776, November 2004.

[4] A. Sahai and D. Cabric, "Spectrum sensing: fundamental limits and practical challenges," in Proceedings of the 1st IEEE International Symposium on New Frontiers in Dynamic Spectrum Access Networks (DySPAN '05), November 2005.

[5] H. Sun, D. I. Laurenson, J. S. Thompson, and C.-X. Wang, "A novel centralized network for sensing spectrum in cognitive radio," in Proceedings of IEEE International Conference on Communications (ICC '08), pp. 4186-4190, May 2008.

[6] W. Zhang, R. K. Mallik, and K. B. Letaief, "Cooperative spectrum sensing optimization in cognitive radio networks," in Proceedings of IEEE International Conference on Communications (ICC'08), pp. 3411-3415, May 2008.

[7] M. R. Musku and P. Cotae, "Time domain spectrum allocation using game theory for cognitive radios," in Proceedings of the 9th IFIP International Conference on Mobile and Wireless Communications Networks (MWCN '07), pp. 101-105, September 2007.

[8] H. Zamat and B. Natarajan, "Use of dedicated broadband sensing receiver in cognitive radio," in Proceedings of IEEE International Conference on Communications (ICC '08), pp. 508-512, May 2008.

[9] The Federal Communications and Commissions, FCC 04113 IEEE 802.22 Working Group on Wireless Regional Area Networks, IEEE P802.22 TM /D0.1, May 2006.

[10] ITU Recommendation G.711, "Pulse Code Modulation (PCM) of Voice Frequencies,” ITU, November 1988.

[11] ITU Recommendation G.726, “40, 32, 24, 16 kbit/s Adaptive Differential Pulse Code Modulation (ADPCM)," ITU, December 1990.

[12] ITU Recommendation G.723.1, "Speech Coders: Dual Rate Speech Coder for Multimedia Communications Transmitting at 5.3 and $6.3 \mathrm{kbit} / \mathrm{s}$," ITU, March 1996.

[13] P. De and Y.-C. Liang, "Blind spectrum sensing algorithms for cognitive radio networks," IEEE Transactions on Vehicular Technology, vol. 57, no. 5, pp. 2834-2842, 2008.

[14] N. Sai Shankar, "Overview of blind sensing techniques considered in IEEE 802.22 WRANs," in Proceedings of the 5th Annual IEEE Communications Society Conference on Sensor, Mesh and Ad Hoc Communications and Networks Workshops (SECON '08), pp. 1-4, June 2008.

[15] G. Ganesan and Y. Li, "Cooperative spectrum sensing in cognitive radio-part I: two user networks," IEEE Transactions on Wireless Communications, vol. 6, no. 6, pp. 2204-2213, 2007.

[16] M. Matsui, H. Shiba, K. Akabane, and K. Uehara, "A cooperative sensing technique with weighting based on distance between radio stations," in Proceedings of the 14th Asia-Pacific Conference on Communications (APCC '08), pp. 1-4, October 2008.

[17] B. Wild and K. Ramchandran, "Detecting primary receivers for cognitive radio applications," in Proceedings of the 1st IEEE International Symposium on New Frontiers in Dynamic Spectrum Access Networks (DySPAN '05), pp. 124-130, November 2005.

[18] T. Yücek and H. Arslan, "Spectrum characterization for opportunistic cognitive radio systems," in Proceedings of IEEE Military Communications Conference (MILCOM '06), pp. 1-6, Washington, DC, USA, October 2006. 
[19] J.-K. Lee, J.-H. Yoon, and J.-U. Kim, "A new spectral correlation approach to spectrum sensing for 802.22 WRAN system," in Proceedings the International Conference on Intelligent Pervasive Computing (IPC '07), pp. 101-104, October 2007.

[20] P. Duhamel, "Algorithms meeting the lower bounds on the multiplicative complexity of length- $2^{\mathrm{n}}$ DFT's and their connection with practical algorithms," IEEE Transactions on Acoustics, Speech, and Signal Processing, vol. 38, no. 9, pp. 1504-1511, 1990.

[21] L. Luo and S. Roy, "Analysis of search schemes in cognitive radio," in Proceedings of the 4th Annual IEEE Communications Society Conference on Sensor, Mesh and Ad Hoc Communications and Networks (SECON '07), pp. 647-654, June 2007.

[22] F. Gardner, Phaselock Techniques, John Wiley \& Sons, New York, NY, USA, 2nd edition, 1979.

[23] V. C. Raykar, R. Duraiswani, and B. Krishnapuram, "A fast algorithm for learning a ranking function from large-scale data sets," IEEE Transactions on Pattern Analysis and Machine Intelligence, vol. 30, no. 7, pp. 1158-1170, 2008. 